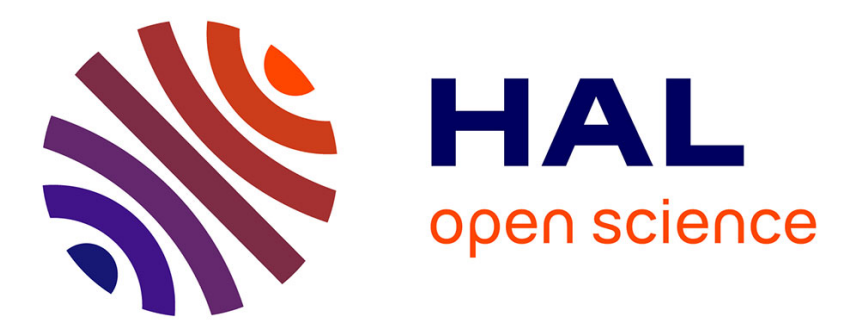

\title{
Multidimensional Pigou-Dalton Transfers and Social Evaluation Functions
}

Marcello Basili, Paulo Casaca, Alain Chateauneuf, Maurizio Franzini

\section{To cite this version:}

Marcello Basili, Paulo Casaca, Alain Chateauneuf, Maurizio Franzini. Multidimensional Pigou-Dalton Transfers and Social Evaluation Functions. 2016. halshs-01321802

\section{HAL Id: halshs-01321802 \\ https://shs.hal.science/halshs-01321802}

Submitted on 26 May 2016

HAL is a multi-disciplinary open access archive for the deposit and dissemination of scientific research documents, whether they are published or not. The documents may come from teaching and research institutions in France or abroad, or from public or private research centers.
L'archive ouverte pluridisciplinaire HAL, est destinée au dépôt et à la diffusion de documents scientifiques de niveau recherche, publiés ou non, émanant des établissements d'enseignement et de recherche français ou étrangers, des laboratoires publics ou privés. 


\section{Documents de Travail du

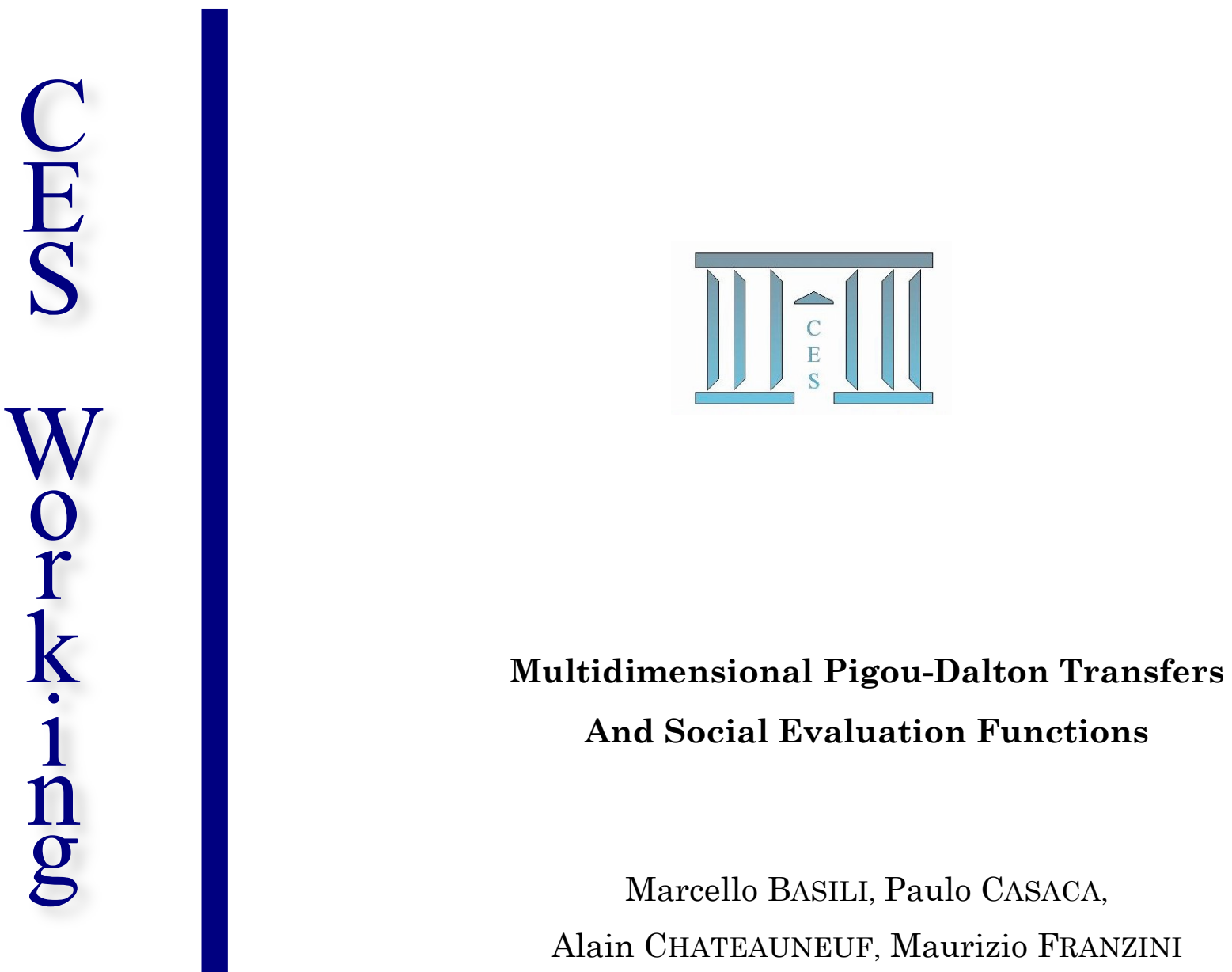

2016.43

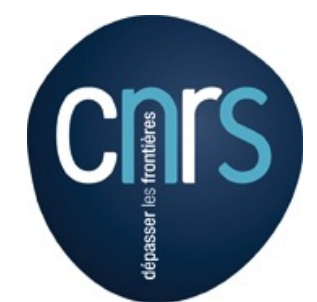




\title{
Multidimensional Pigou-Dalton Transfers And Social Evaluation Functions
}

\author{
Marcello Basili \\ DEPS and SEM, University of Siena \\ Paulo Casaca* \\ UFMG, Universidade Federal de Minas Gerais \\ Alain Chateauneuf ${ }^{\dagger}$ \\ IPAG Business School and PSE-CES, University of Paris-I \\ Maurizio Franzini \\ DIPECODIR, University of Rome La Sapienza
}

\begin{abstract}
We axiomatize, in the multidimensional case, a social evaluation function that can accommodate a natural Pigou-Dalton principle and correlation increasing majorization. This is performed by building upon a simple class of inframodular functions proposed by Müller and Scarsini under risk.

Keywords: multidimensional inequality, Pigou-Dalton transfer, increasing majorization, inframodular functions, Human Development Index.
\end{abstract}

*Corresponding Author. Université Paris 1 Panthéon-Sorbonne. 106 - 112 Boulevard de l'Hôpital, 75013 Paris, France; e-mail: paulorscasaca@yahoo.com.br

$\dagger$ Alain Chateauneuf gratefully acknowledges the hospitality and the support of the University of Rome La Sapienza, during his stay as visiting professor in Spring 2014. 


\section{Introduction}

There has been a resurgence of interest in multidimensional social evaluation functions mainly due to new techniques that extend in the multidimensional setting the pioneering works by Atkinson (1970; 1987), Kolm (1976a;b; 1977) and Sen (1976). In particular, Tsui $(1995 ; 1999)$ and Gajdos and Weymark (2005) have offered axiomatic approaches to designing income inequality measures in a multiattribute context.

Although Tsui mainly used the additive approach, Gajdos and Weymark built upon the generalized Gini social function. These two different approaches are not at all innocuous. The former aggregates the attributes of each individual and then additively aggregates the resulting values; the latter evaluates the different attributes through a specific aggregation and then simply aggregates the values.

Note also that although Tsui's (1999) approach is a 'traditional' additive evaluation, Gajdos and Weymark (2005) adopted a non-additive approach, which was introduced by Weymark in his seminal paper in 1981 .

In this paper, we follow the traditional additive approach, but instead of imposing the majorization theory of the m-dimensional case as in Tsui (1995), we confine ourselves to accommodating a particular Pigou-Dalton transfer, which we believe is relevant. Furthermore our approach is consistent with the meaningful property of correlation increasing majorization (e.g. Tsui, 1999).

The paper is organized as follows. Section 2 analyzes the related literature. Section 3 presents our motivations. Section 4 states the framework and the axioms. Section 5 offers our main Theorem, specifically the Theorem 2 which characterizes our social evaluation function. Section 6 aims at reducing the number of parameters, namely, at specifying some fundamental $\psi$ function, for our purpose. Thus we deliver a tractable relative inequality index in the Corollary of Theorem 3. Section 7 shows that our evaluation function can accommodate correlation increasing majorization. Section 8 evaluates the appliance of our inframodular social evaluation, by comparing it with the famous Human Development Index (HDI) by using effective data. Section 9 concludes and proofs can be found in the Annex.

\section{Related literature}

In the last two decades, consensus has been emerging among many scientists, particularly economists, about the multidimensional aspect of individual wellbeing that cannot be reduced to a unique monetary dimension. The origin of the multidimensional approach to poverty and/or inequality can be traced to the works of Rawls and Sen on ethical principles. In this perspective, as an example, health and education are important dimensions of individual well-being to be considered along with income. Crucially, many additional aspects of wellbeing may not be compared on a true cardinal basis; nonetheless, the literature assumes that all attributes have a cardinal meaning (Allison and Foster, 2004). 
Unidimensional methods of evaluating poverty and inequality are applied when a single well-defined dimensional variable, i.e., income, has been selected. The selected variable can be cardinal, more often, or ordinal, and in this unidimensional environment, rank is defined regarding the minimum level, named the poverty line, below which a person is considered poor. If the assumed notion of poverty (inequality) is considered a useful proxy (unidimensional lens) that emerges after taking into account (and merging) different dimensions, then a unidimensional methodology is coherent and consistent.

On the contrary, if an aggregate variable cannot be constructed because there is a set of non-cardinal and non-summable attributes (health, education, talent, capabilities, etc.) in addition to income or consumption, then identification and measurement problems emerge. Identification means, for example, that is necessary to define the conditions under which a person is considered poor, and a measure must evaluate how much poverty there is overall (Sen, 1976). Poverty and inequality, like development, are multidimensional. In the economic literature, for ranking individuals who differ in attributes, there are two main approaches: The first uses a social welfare function (i.e. Atkinson and Bourguignon, 1982); the second extends the Foster et al. (1984) class of indices (decomposable poverty measures based on powers of normalized shortfalls) by using inequality statistics and measuring individual attributes with a utility function, so that the resulting univariate distribution vector of utilities is valued through an inequality index. Atkinson and Bourguignon (1982) attach to the attributes a symmetrical role; in fact, they introduce the crucial idea of complementarity or substitutability between attributes, which may be expressed as a social taste either for increasing correlation or for decreasing correlation. They study the correspondence between stochastic dominance conditions and the welfare interpretation of the value judgments. Interesting enough, comparing distributions according either to an additive separable social welfare function with concave utilities or to the sequence of Pigou-Dalton progressive transfers leads to the same conclusion as resorting to Lorenz curves or computing the amount of aggregate poverty gaps. In a seminal paper, Maasoumi (1986) introduces a two-stage approach for defining the class of generalized entropy measures.

More interesting for our research, in 1995 Tsui introduces a multidimensional generalization of the Atkinson-Kolm-Sen approach to measure inequality. Tsui defines a set of axioms "which are generalizations of their counterparts in the unidimensional context and are often considered to have a higher degree of acceptability." (1995; p. 254). In this way, he distinguishes absolute and relative multidimensional equality indices based on a social evaluation function. Gajdos and Weymark (2005) follow Tsui's general approach and obtain an univariate distribution vector of utilities that is valued using an inequality index. In particular, Gajdos and Weymark extend generalized Gini social evaluation functions to the multivariate case with a comonotonic independence axiom, such that "two allocations are said to be comonotonic if all individuals are ranked identically in all attributes (i.e., the richest is also the most educated etc.), and the ranking between two comonotonic allocations is not reversed by the addition of a comonotonic allocation." (Galichon and Henry, 2012; p. 1513). In 2006, 
Duclos et al. showed that it is possible to make sensible comparisons of poverty when accounts for multiple dimensions of well-being and that multidimensional comparisons can also differ from univariate comparisons in each individual dimension. More recently, Müller and Scarsini (2012) introduced inframodular utility functions that represent the attitude of an agent who dislikes transfers that move mass from inside a multidimensional interval to the sets above and below it, mimicking the mean preserving spread. Since a function is concave if and only if it has non-increasing differences, in the same way for multivariate functions, inframodularity (generalized concavity) implies that there are nonincreasing differences. In this way, Müller and Scarsini use inframodular functions to model risk aversion that involves substitutable, but not complementary, commodities.

\section{Main motivation}

In the unidimensional case, when considering $n$ individuals $1, \ldots, j, \ldots, n$ with incomes $x_{1} \leq x_{2} \leq \ldots \leq x_{j} \leq \ldots \leq x_{n}$, it is usually assumed that if $x_{j}<x_{j+1}$ then a transfer $\varepsilon>0$ from individual $j+1$, to individual $j$ such that $x_{j}+\varepsilon \leq$ $x_{j+1}-\varepsilon$ reduces inequality. This transfer is a Pigou-Dalton transfer. Note that this is equivalent to assuming that if $x_{j} \leq x_{j+1}$, then modifying $x_{j}$ into $x_{j}-\varepsilon$ and $x_{j+1}$ into $x_{j+1}+\varepsilon$ increases inequality.

Thus turning to the $m$-dimensional case $m \geq 1$, which is the topic of this paper, where each individual $j$ has a column-vector $A_{j} \in \mathbb{R}^{m}$ of $m$ attributes, we get that the $m \times n$ matrix $A=\left(A_{1}, \ldots, A_{j}, \ldots, A_{n}\right)$ summarizes the data. Imagine that for two individuals their respective column attributes are $X$ and $Y$, with $X \leq Y$ (i.e. $x_{i} \leq y_{i}, \forall i=1, \ldots, m$ ) and let $\varepsilon \in \mathbb{R}_{+}^{m}$ with $\varepsilon \neq 0$. In such a situation, transfer $\varepsilon$ from $X$ to $Y$ will be a regressive Pigou-Dalton transfer because it would increase inequalities. ${ }^{1}$

Our goal is to axiomatize additive social evaluation functions, i.e., social evaluation functions $I: A \longrightarrow \mathbb{R}$ such that $I(A)=\sum_{j=1}^{n} u\left(A_{j}\right)$ where $u: \mathbb{R}^{m} \longrightarrow$ $\mathbb{R}$ agrees with a diminishing social evaluation in the case of such a Pigou-Dalton regressive transfer as above. It is immediate that $\forall(X, Y) \in \mathbb{R}^{m} \times \mathbb{R}^{m}$ such that $X \leq Y$ and $\forall \varepsilon \geq 0, u$ should satisfy:

$$
u(X)-u(X-\varepsilon) \geq u(Y+\varepsilon)-u(Y)
$$

This is the usual property of concavity in the one-dimensionalcase, at least when $u$ is continuous. Actually, we show (see the Annex) that inframodular functions, extensively studied by Marinacci and Montrucchio (2005) and proposed by Müller and Scarsini (2001) as a meaningful representation of risk

\footnotetext{
${ }^{1}$ Since this paper has been performed, we have been aware of a similar Pigou-Dalton principle introduced by Bosmans et al. (2009). Nevertheless main differences persist between the two papers: our definition is model-free and our main motivation is to link this principle to inframodularity.
} 
aversion in the multidimensional case, satisfy this desired property (1), and are consistent with the relevant property of correlation increasing majorization. Inframodular functions may not be concave (e.g. Marinacci and Montrucchio, 2005); therefore, as observed by Müller and Scarsini (2001), inframodular functions do not match the property of risk aversion, which states that adding a random vector $E$ with mean 0 to a constant multivariate vector is always unfavorable. Note also that multidimensional concave functions may not be inframodular. A typical example is the three-dimensional HDI function $v:\left(x_{1}, x_{2}, x_{3}\right) \in[0,1]^{3} \longrightarrow v\left(x_{1}, x_{2}, x_{3}\right)=x_{1}^{\frac{1}{3}} x_{2}^{\frac{1}{3}} x_{3}^{\frac{1}{3}}$. Therefore, an additive social evaluation function based on HDI might not respect the natural multidimensional Pigou-Dalton principle evocated above. As for an example let us consider two individuals with row attributes respectively $X=(0.8,0.64,0.729)$ and $Y=(0.9,0.81,0.729)$ and let us transfer $\varepsilon=(0,0,0.1)$ from $X$ to $Y$. A simple computation delivers $v(X-\varepsilon)+v(Y+\varepsilon)>v(X)+v(Y)$.

Indeed, the updated $\mathrm{HDI}^{2}$ denoted $v$ above would not value the two previous situations as in the former computation, but the ranking would be again disputable since:

$$
H D I(X, Y)=v\left(\frac{X+Y}{2}\right)=v\left(\frac{X-\varepsilon+Y+\varepsilon}{2}\right)=H D I(X-\varepsilon, Y+\varepsilon)
$$

As a consequence this index would not take into account what appears as a clear deterioration of the social function with respect to inequality, when modifying $(X, Y)$ into $(X-\varepsilon, Y+\varepsilon)$.

This contrasts with the additive social evaluation function that we propose in this paper (e.g. Theorem 3 and Section 8), namely $I(X, Y)=u(X)+u(Y)$ where $u\left(x_{1}, x_{2}, x_{3}\right)=\ln \frac{x_{1}+x_{2}+x_{3}}{3}$ which delivers,

$$
I(X, Y)=\ln (0.587)>I(X-\varepsilon, Y+\varepsilon)=\ln (0.583)
$$

hence a ranking in accordance with the intuition.

As pointed out in the introduction, we intend to test the pertinence of inframodular social evaluations when compared to the famous HDI. This explains why we will focus on particularly tractable and meaningful inframodular functions $u$ as proposed by Müller and Scarsini (2001; 2012) through:

$$
u\left(x_{1}, \ldots, x_{i}, \ldots, x_{m}\right)=\psi\left(\sum_{i=1}^{m} \alpha_{i} x_{i}\right)
$$

where $\psi$ is concave and $\alpha_{1}, \ldots, \alpha_{m} \geq 0$.

Note that such an $u$ is a valuable function for our purpose, since, first, it makes sense to weight the different attributes in accordance with their importance $\alpha_{i} \geq 0$, and second, with such a $\psi$ concave, the resulting $u$ is inframodular, so it agrees with our definition of increasing inequality.

\footnotetext{
${ }^{2}$ In 2010 the HDI functional has changed its additive form to a multiplicative form as mentioned above. Section 8 includes a discussion about this 'new' HDI. Details are in Zambrano (2014).
} 
Accordingly, in this paper, we mainly axiomatize the social evaluation function of the type given by (2), present the natural usual axioms aiming to specify $\psi$, and propose a simple relative inequality index. Moreover, as pointed out in the Introduction, our social evaluation functions is proved to satisfy the condition of correlation increasing majorization.

\section{Framework and axioms}

We consider $n$ individuals $1, \ldots, j, \ldots, n$ and $A_{j} \in \mathbb{R}^{m}$ is the column-vector of the $m$ attributes of this individual $A_{j}=\left(\begin{array}{c}a_{1 j} \\ \cdot \\ a_{i j} \\ \cdot \\ a_{m j}\end{array}\right)$; indeed, the same $m$ attributes are considered for each individual. ${ }^{3}$

Henceforth, for $n$ given individuals, $A=\left(A_{1}, \ldots, A_{j}, \ldots, A_{n}\right)$ is the $m \times n$ matrix summarizing the considered population.

$\mathcal{A}$ denotes the set of real matrices. If $a_{i j} \geq 0 \forall i$ and $\forall j$, we use the notation $\mathcal{A}_{+}$, and if $a_{i j}>0 \forall i$ and $\forall j$, we use the notation $\mathcal{A}_{++}$.

Thus $\succsim$ is a preference relation on $\mathcal{A}$ (if $\mathcal{A}_{+}$or $\mathcal{A}_{++}$is used, this is specified in the Theorems). Here, we present the version of the axioms for $\mathcal{A}$. Indeed, for $A, B \in \mathcal{A}, A \succsim B$ means $A$ is weakly preferred to $B$, etc.

Then $\succsim$ is supposed to express the preferences of the policy-maker or the modeler for global welfare, taking into account that inequalities have a bad impact on welfare, but also that all attributes are 'positive'; that is, any increase in some attribute has a positive effect on welfare.

The first three axioms are standard; therefore, they do not require a particular explanation:

$A .1 \succsim$ is a weak order; i.e., $\succsim$ is a transitive, complete hence reflexive binary relation on $\mathcal{A}$.

A.2 Continuity: Let $B \in \mathcal{A}$ be given, then $\{A \in \mathcal{A} \mid A \succsim B\}$ and $\{A \in \mathcal{A} \mid A \preceq B\}$ is closed in the usual topology of $\mathbb{R}^{n \times m}$.

A.3 Monotonicity: $\forall A, B \in \mathcal{A}, a_{i j} \geq b_{i j} \forall i, j$, implies $A \succsim B$; if furthermore $A \neq B$, then $A \succ B$.

For $A \in \mathcal{A}$ and $A_{j}^{\prime}$ a column of $\mathbb{R}^{m},\left(A_{j}^{\prime}, A_{-j}\right)$ denotes the matrix $A$ where column $A_{j}$ has been replaced by column $A_{j}^{\prime}$. Thus, the classical independence axiom that states that the impact for the ranking of replacing a given individual by another one is the same if all the other individuals remain unchanged.

A.4 Independence: $\forall j$ and $\forall A, B\left(A_{j}, A_{-j}\right) \succsim\left(A_{j}^{\prime}, A_{-j}\right) \Longleftrightarrow\left(A_{j}, B_{-j}\right) \succsim$ $\left(A_{j}^{\prime}, B_{-j}\right)$.

Below is the classical anonymity index that states that the value of a distribution does not depend on the identity; only the value of the attributes matters.

\footnotetext{
${ }^{3}$ Note that throughout the paper we assume $n \geq 3$.
} 
A.5 Anonymity: for any permutation matrix $\Pi$ and for all $A \in \mathcal{A}$, one has $A \sim \Pi A$; i.e., $\left(A_{1}, \ldots, A_{j}, \ldots, A_{n}\right) \sim\left(A_{\sigma(1)}, \ldots, A_{\sigma}\left({ }_{j}\right), \ldots, A_{\sigma(n)}\right)$ where $\sigma:[1, n] \rightarrow[1, n]$ is a bijection.

The last two axioms, to the best of our knowledge, are new and are crucial for our purpose.

Roughly speaking, the additivity axiom states that if an individual with a given vector of attributes is indifferent between two different lists of attributes for the others, he remains indifferent if he is endowed with a new vector of attributes.

A.6 Additivity: $\forall A, A_{j}, B_{j}, C_{j},\left(A_{j}, A_{-j}\right) \sim\left(B_{j}, A_{-j}\right) \Longrightarrow\left(A_{j}+C_{j}, A_{-j}\right) \sim$ $\left(B_{j}+C_{j}, A_{-j}\right)$.

The following Pigou-Dalton principle is the direct translation of the fact that if for an individual $\left(j_{1}\right)$ all the attributes are smaller than for another one $\left(j_{2}\right)$, then transferring for any $i$ a value $\varepsilon_{i} \geq 0$ of attribute $i$ from $j_{1}$ to $j_{2}$ clearly should increase the inequality (strictly increase if some $\varepsilon_{i}>0$ ), thus leading to a worse situation.

A.7 Pigou-Dalton principle: Let $A=\left(A_{1}, \ldots, A_{j}, \ldots, A_{n}\right)$ such that for some $j_{1}, j_{2}$ one has $A_{j_{1}} \leq A_{j_{2}}$ and let $\varepsilon \in \mathbb{R}_{+}^{m}$ then:

$A_{\varepsilon}=\left(A_{1}, \ldots, A_{j_{1-1}}, A_{j_{1}}-\varepsilon, A_{j_{1+1}}, \ldots, A_{j_{2-1}}, A_{j_{2}}+\varepsilon, A_{j_{2+1}}, \ldots, A_{n}\right) \precsim A ;$ furthermore, $A_{\varepsilon} \prec A$ if $\varepsilon \geq 0$ but $\varepsilon \neq 0$.

\section{Multidimensional social evaluation functions}

In this Section, multidimensional social evaluation functions are defined and characterized. Theorem 1 offers axiomatization of the additive social evaluation function.

Theorem 1 A preference relation $\succsim$ on $\mathcal{A}$ satisfies A.1, A.2, A.3, A.4, A.5 if and only if there exists: $u: \mathbb{R}^{m} \longrightarrow \mathbb{R}$ increasing and continuous satisfying (1), such that:

$$
\forall A, B \in \mathcal{A}, A \succsim B \Longleftrightarrow \sum_{j=1}^{n} u\left(A_{j}\right) \geq \sum_{j=1}^{n} u\left(B_{j}\right)
$$

where $u$ is defined up to a positive affine transformation (the proof is in the Appendix).

We come now to the main result of this paper in which we characterize social evaluation functions built upon the special type of inframodular functions satisfying (2) as proposed in a different framework by Müller and Scarsini. Such a social function agrees with our Pigou-Dalton principle $A .7$ and with the property of correlation increasing majorization as shown in Section $\%$.

Theorem 2 A preference relation on $\mathcal{A}$ satisfies A.1, A.2, A.3, A.4, A.5, A.6 and $A .7$ if and only if there exist $\alpha_{i}>0, i=1, \ldots, m$, such that $\sum_{i=1}^{m} \alpha_{i}=1$ and 
there exists $\psi: \mathbb{R} \rightarrow \mathbb{R}$ strictly increasing, strictly concave and continuous such that:

$$
A \succsim B \Longleftrightarrow \sum_{j=1}^{n} \psi\left(\sum_{i=1}^{m} \alpha_{i} \cdot a_{i j}\right) \geq \sum_{j=1}^{n} \psi\left(\sum_{i=1}^{m} \alpha_{i} \cdot b_{i j}\right) .
$$

Furthermore, such $\alpha_{i}^{\prime}$ s are unique and $\psi$ is defined up to an increasing affine transformation.

Proof. The necessary part of the proof is straightforward since inframodular functions satisfy A.7 (see Lemma 1 in the Annex); thus we confine ourselves to proving the sufficiency part of the proof. From Theorem 1, we already know that the preference relations $\succeq_{j}$ on $\mathbb{R}^{m}$ of every individual $j$ are identical.

Let us denote $\stackrel{*}{\succsim}$ this common preference relation and let us show that there exists $\alpha_{i}>0, i=1, \ldots, m$, which $\sum_{i=1}^{m} \alpha_{i}=1$ such that $\forall(X, Y) \in \mathbb{R}^{m} \times \mathbb{R}^{m}$,

$$
X \stackrel{*}{\succsim} Y \Longleftrightarrow \sum_{i=1}^{m} \alpha_{i} x_{i} \geq \sum_{i=1}^{m} \alpha_{i} y_{i} .
$$

Moreover, A.1, A.2, A.3, A.4 imply that $\stackrel{*}{\succsim}$ satisfies:

$A^{*} .1: \stackrel{*}{\succsim}^{*}$ is a weak order;

$A^{*}$.2: continuity: $X^{(p)}, X, Y \in \mathbb{R}^{m}$ then:

$A^{*} .2 .1: X^{(p)} \stackrel{*}{\succsim} Y, \forall p, \quad X^{(p)} \downarrow X \Longrightarrow X \stackrel{*}{\succsim} Y$

$A^{*}$.2.2: $X^{(p)} \stackrel{*}{\precsim} Y, \forall p, X^{(p)} \uparrow X \Longrightarrow X \stackrel{*}{\precsim} Y$

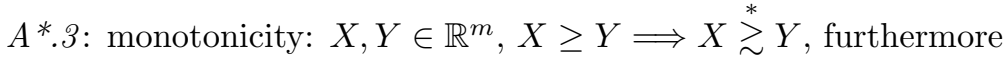

if $X \neq Y \Longrightarrow X \stackrel{*}{\succ} Y$

$A^{*}$.4: additivity: $\forall X, Y, Z \in \mathbb{R}^{m}, X \stackrel{*}{\sim} Y \Longrightarrow X+Z \stackrel{*}{\sim} Y+Z$

For $X \in \mathbb{R}^{m}$ denote $I(X): \equiv \operatorname{Inf}\{x \mathbb{R} x \cdot 1 \stackrel{*}{\succsim} X\}$, it is easy to see that

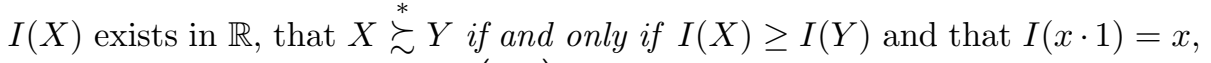
$\forall x \in \mathbb{R}$, where indeed $1=\left(\begin{array}{c}1 \\ \cdot \\ \cdot \\ \cdot \\ 1\end{array}\right) \in \mathbb{R}^{m}$

Let $e_{i}$ be the $i_{t h}$ vector of the canonical basis of $\mathbb{R}^{m}$, i.e., $e_{i}=\left(\begin{array}{c}0 \\ \dot{1} \\ 1 \\ 0\end{array}\right)$ the $i_{\text {th }}$ row, and let $\alpha_{i}: \equiv I\left(e_{i}\right)$, from $A^{*}$.3, since $I(0)=0$, one gets $\alpha_{i}>0$.

We now intend to show that 
(3)

$$
I(X)=\sum_{i=1}^{m} \alpha_{i} x_{i}
$$

Note that since $I(1)=1$ this will entail $\sum_{i=1}^{m} \alpha_{i}=1$.

In order to prove (3), let us show first that: $\forall Y, Z \in \mathbb{R}^{m}$ one has

(4) $\quad I(Y+Z)=I(Y)+I(Z)$

Since $Y \stackrel{*}{\sim} I(Y) \cdot 1$ and $Z \stackrel{*}{\sim} I(Z) \cdot 1 \mathrm{~A}^{*} .4$ implies (4), since $Y+Z \stackrel{*}{\sim} I(Y) \cdot 1+Z$ and $I(Y) \cdot 1+Z \stackrel{*}{\sim}(I(Y)+I(Z)) \cdot 1$, gives $Y+Z \stackrel{*}{\sim}(I(Y)+I(Z)) \cdot 1$

It turns out that $I(X)=\sum_{i=1}^{m} I\left(x_{i} \cdot e_{i}\right)$. It remains to show that $I\left(x_{i} \cdot e_{i}\right)=$ $x_{i} I\left(e_{i}\right)$.

It is enough to prove that.

(5) $\quad I(x \cdot X)=x \cdot I(X) \forall x \in \mathbb{R}$ and $\forall X \in \mathbb{R}^{m}$.

This has been already proved for $x=0$. So let us assume $x \in \mathbb{R}^{*}$.

Assume first $x \in \mathbb{Q}^{*}$ i.e. $x=\frac{p}{q}, p \in \mathbb{N}^{*}, q \in \mathbb{Z}^{*}$.

From (4) $I\left(\frac{p}{q} \cdot X\right)=I\left(p \cdot \frac{X}{q}\right)=p \cdot I\left(\frac{X}{q}\right)$ but $I(X)=I\left(q \cdot \frac{X}{q}\right)=q \cdot I\left(\frac{X}{q}\right)$

Therefore, $I\left(\frac{p}{q} \cdot X\right)=\frac{p}{q} \cdot I(X)$.

From A*.2 it is simple to see that $X_{n} \downarrow X \Longrightarrow I\left(X_{n}\right) \downarrow I(X)$ and

$X_{n} \uparrow X \Longrightarrow I\left(X_{n}\right) \uparrow I(X)$, and that $I$ is monotone i.e. $X \geq Y \Longrightarrow I(X) \geq$ $I(Y)$.

So let us consider now $x \in \mathbb{R}$ and $x_{n} \in \mathbb{Q}, x_{n} \downarrow x, y_{n} \in \mathbb{Q}, y_{n} \uparrow x$, from $\mathrm{A}^{*} .3$ $x_{n} \cdot X \geq x \cdot X \geq y_{n} \cdot X$ implies:

$x_{n} \cdot X \stackrel{*}{\succsim} x \cdot X \stackrel{*}{\succsim} y_{n} \cdot X \quad$ so $I\left(x_{n} \cdot X\right) \geq I(x \cdot X) \geq I\left(y_{n} \cdot X\right)$

Therefore, $x_{n} \cdot I(X) \geq I(x \cdot X) \geq y_{n} \cdot I(X) \forall n$, so letting $n \longrightarrow+\infty$,

we get $I(x \cdot X)=x \cdot I(X)$, which completes the fact that:

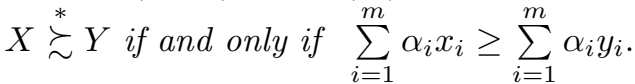

We end the proof by showing that up to an increasing affine transformation there exists a unique $\psi: \mathbb{R} \rightarrow \mathbb{R}$ strictly increasing, strictly concave, and continuous such that $\forall A, B \in \mathcal{A}$ :

$A \succsim B \Longleftrightarrow \sum_{j=1}^{n} \psi\left(\sum_{i=1}^{m} \alpha_{i} \cdot a_{i j}\right) \geq \sum_{j=1}^{n} \psi\left(\sum_{i=1}^{m} \alpha_{i} \cdot b_{i j}\right)$.

From Theorem 1 there exists - up to a positive affine transformation - a unique $u$ increasing and continuous satisfying (1) such that:

$X, Y \in \mathbb{R}^{m}, X \stackrel{*}{\succsim} Y \Longleftrightarrow u(X) \geq u(Y)$.

Since $I: \mathbb{R}^{m} \rightarrow \mathbb{R}$ is also a strictly increasing and continuous representation of $\stackrel{*}{\succsim}$, there exists up to a positive affine transformation a strictly increasing continuous function $\psi: \mathbb{R} \rightarrow \mathbb{R}$ such that $u=\psi \circ I$.

It remains to be proved that $\psi$ is strictly concave.

It is enough to show that $\forall(a, b) \in \mathbb{R}^{2}, a \leq b, \forall \varepsilon>0$ one has $\psi(a)-\psi(a-\varepsilon)>$ $\psi(b+\varepsilon)-\psi(b)$.

It is immediate to find $X, Y \in \mathbb{R}^{m}$ such that $X \leq Y$ and 


$$
\begin{aligned}
\sum_{i=1}^{m} \alpha_{i} x_{i}=a, b= & \sum_{i=1}^{m} \alpha_{i} y_{i}, \text { so from } A .7, \text { we get } \\
& u(X)-u(X-\varepsilon \mathbb{1})>u(Y+\varepsilon \mathbb{1})-u(Y)
\end{aligned}
$$

Therefore, $\psi(a)-\psi(a-\varepsilon)>\psi(b+\varepsilon)-\psi(b)$, which completes the proof of Theorem 2

\section{Specification of $\psi$ and a relative inequality in- dex}

To specify $\psi$, we introduce two axioms that have a long tradition; see, for instance, Kolm (1976a;b) and, more recently, Gajdos and Weymark (2005).

$A .8 \forall A, B \in \mathcal{A}$ and $\forall \lambda \in \mathbb{R}, A \sim B \Longleftrightarrow A+\lambda \mathbb{1} \sim B+\lambda \mathbb{1}$ where $\mathbb{1}$ is the matrix $m \times n$ with 1 everywhere.

This axiom quoted as a 'leftist' point of view by Kolm regarding inequalities expresses that the inequalities remain unchanged if the same amount is added to all attributes and to all individuals.

The following axiom quoted as a 'centrist' point of view by Kolm regarding inequalities, and that applies only if all the attributes are strictly positive, i.e., $A \in \mathcal{A}_{++}$, expresses that inequalities remain unchanged if all attributes are multiplied by the same positive number $\lambda>0$ for all individuals.

$A .9 \forall A, B \in \mathcal{A}_{++}$and $\forall \lambda>0, A \sim B \Longleftrightarrow \lambda A \sim \lambda B$

Theorem 3 Assume that the preference relation $\succsim$ on $\mathcal{A}$ satisfies A.1 to A.7, then:

- up to an increasing affine transformation $\psi(t)=-e^{-a t}$ with $a>0$ if and only if A.8 is satisfied;

- up to an increasing affine transformation either $\psi(t)=\ln (t), \forall t>0$ or $\psi(t)=t^{a}, \forall t>0$ where $a \neq 0, a<1$ if and only if A.9 is satisfied.

Proof. We prove only the if part; the only if partis straightforward.

Assume $A .8$ is satisfied. It is easy to see that if $\sum_{j=1}^{n} \psi\left(x_{j}\right)=\sum_{j=1}^{n} \psi\left(y_{j}\right)$ where $x_{j}, y_{j} \in \mathbb{R}$, we must have $\sum_{j=1}^{n} \psi\left(x_{j}+k\right)=\sum_{j=1}^{n} \psi\left(y_{j}+k\right) \quad \forall k \in \mathbb{R}$, and then we can apply the results of the classical one-dimensional social welfare theory (see e.g. Kolm, 1976a) to get the desired result.

Assume A.9 is satisfied. It is easy to see that $\sum_{j=1}^{n} \psi\left(x_{j}\right)=\sum_{j=1}^{n} \psi\left(y_{j}\right)$ where $x_{j}>0, y_{j}>0, \forall j$ implies $\sum_{j=1}^{n} \psi\left(\lambda x_{j}\right)=\sum_{j=1}^{n} \psi\left(\lambda y_{j}\right) \quad \forall \lambda>0$, and then we can apply the results of the classical one-dimensional social welfare theory ${ }^{4}$

\footnotetext{
${ }^{4}$ See e.g. Kolm (1976a;b) or Atkinson (1970) to get the result
} 
Remark Note that in cases in which all attributes are strictly positive, and if we adopt axiom $A .9$ then $\forall A \in \mathcal{A}_{++}$one could adopt the social evaluation function $J(A)=\prod_{j=1}^{n}\left(\sum_{i=1}^{m} \alpha_{i} \cdot a_{i j}\right)$.

Indeed, in such a case: $I(A)=\sum_{j=1}^{n} \ln \left(\sum_{i=1}^{m} \alpha_{i} \cdot a_{i j}\right)=\ln \left(\prod_{j=1}^{n}\left(\sum_{i=1}^{m} \alpha_{i} \cdot a_{i j}\right)\right)$.

We focus on the relative inequality index that is linked with the choice of $\psi()=.\ln ($.$) . This index appears to be one of the most tractable and relevant in$ our framework.

\subsection{Corollary of Theorem 3}

The corresponding inequality index related to the social evaluation function defined on $\mathcal{A}_{++}$, the set of $m \times n$ matrices with positive elements, satisfying $A .1$ to $A .7$ and $A .9$ with $\psi(t)=\ln (t)$, with $t>0$ is relative and has the form

$$
1-\left(\prod_{j=1}^{n} \frac{\sum_{i=1}^{m} \alpha_{i} \cdot a_{i j}}{\sum_{i=1}^{m} \alpha_{i} \cdot \mu_{i}}\right)^{\frac{1}{n}}
$$

Where $\mu_{i}, i=1, \ldots, m$, is the mean of $i^{t h}$ attribute.

Proof. Following Tsui (1995) and Kolm (1977), let us define the multidimensional inequality index $I_{R}(A)$ for $A \in \mathcal{A}_{++}$as $I_{R}(A)=1-\delta(A)$ where $\delta(A) \in[0,1]$ is defined by $I(A)=I\left(\delta(A) \cdot A_{\mu}\right)$

where $A_{\mu}$ is the $m \times n$ matrix where each column writes $\left(\begin{array}{c}\mu_{1} \\ \cdot \\ \mu_{i} \\ \cdot \\ \mu_{m}\end{array}\right)$. From $I(A)=\sum_{j=1}^{n} \ln \left(\sum_{i=1}^{m} \alpha_{i} \cdot a_{i j}\right)=\ln \prod_{j=1}^{n}\left(\sum_{i=1}^{m} \alpha_{i} \cdot a_{i j}\right)$ and $I(\delta(A))=\ln \left(\delta(A)^{n} \cdot\left(\sum_{i=1}^{m} \alpha_{i} \cdot \mu_{i}\right)^{n}\right)$ one gets the desired result, namely, $I_{R}(A)=1-\left(\prod_{j=1}^{n} \frac{\sum_{i=1}^{m} \alpha_{i} \cdot a_{i j}}{\sum_{i=1}^{m} \alpha_{i} \cdot \mu_{i}}\right)^{\frac{1}{n}}$

\section{Agreeing with correlation increasing majoriza- tion.}

Correlation increasing majorization $(C I M)$ is a concept due to Boland and Proschan (1988) and introduced into the inequality literature by Tsui (1999). As pointed out by Tsui (1999), this type of majorization is known as an ordering of dependence in statistics (e.g. Shaked, 1982) and in economics of risks as 'pairwise more risk' (Richard, 1975). Note that CIM or the majorization axiom corresponds to Atkinson-Bourguignon ordering (Atkinson and Bourguignon, 1982), but Gajdos and Weymark (2005) observed that Bourguignon and Chakravarty (2003) raised reservations about this axiom, because CIM does not take into 
account individual preferences. Since the point of view of our social evaluation is to consider a policy-maker or else a modeler who aims to consider each individual in the same way, we do not concur with the previous reservation and agree with the motivating examples given by Tsui in 1999 .

Let $A, B, C$ be the following three matrices summarizing the distributions of attributes, ${ }^{5}$

$$
A=\left(\begin{array}{lll}
1 & 2 & 3 \\
2 & 3 & 1 \\
3 & 2 & 1
\end{array}\right) \quad B=\left(\begin{array}{lll}
1 & 2 & 3 \\
1 & 3 & 2 \\
1 & 2 & 3
\end{array}\right) \quad C=\left(\begin{array}{lll}
1 & 2 & 3 \\
1 & 2 & 3 \\
1 & 2 & 3
\end{array}\right)
$$

Similar to Tsui (1999), we feel intuitively compelled to agree that the distribution of attributes summarized by $C$ is most unequal followed by $B$ and then by $A$.

Note that column $B_{1}$ and $B_{3}$ are nothing other than $B_{1}=A_{1} \wedge A_{3}$ and $B_{3}=A_{1} \vee A_{3}$, where $\wedge$ and $\vee$ are the classical operators min and max.

Furthermore, $C_{2}=B_{2} \wedge B_{3}$ and $C_{3}=B_{2} \vee B_{3}$.

It is time to define formally correlation increasing majorization.

First, let us introduce some definitions (see Boland and Proschan, 1988).

Concept of correlation increasing transfer (CIT)

Let $A, B \in \mathcal{A}$, then $B$ is obtained from $A$ by a $C I T$ if there exists $j_{1}, j_{2}$, $j_{1} \neq j_{2}$ such that $B_{j_{1}}=A_{j_{1}} \wedge A_{j_{2}}$ and $B_{j_{2}}=A_{j_{1}} \vee A_{j_{2}}$

A $C I T$ is strict whenever neither $A_{j_{1}} \leq A_{j_{2}}$ nor $A_{j_{2}} \leq A_{j_{1}}$.

Concept of correlation increasing majorization (CIM)

Let $A, B \in \mathcal{A}$, then $A>_{c} B$; i.e., $A$ is strictly less unequal for the $C I M$ if $B$ may be derived from $A$ by a permutation of columns and a finite sequence of the correlation increasing transfers at least one of which is strict.

We can now state and prove that our social evaluation functional of Theorem 2 as well as any strict inframodular social functional of Theorem 1 respect CIM.

We say that an inframodular function $u$ is strict if:

$\forall(X, Y) \in \mathbb{R}^{m} \times \mathbb{R}^{m}, X<Y, \quad$ i.e., $\quad X \leq Y, X \neq Y$ and $\varepsilon \geq 0, \varepsilon \in \mathbb{R}^{m}$, $\varepsilon \neq 0$,

one has $u(X+\varepsilon)-u(X)>u(Y+\varepsilon)-u(Y)$

Note this is the case for the inframodular function in Theorem 2.

Theorem 4 Any strict inframodular social evaluation functional respects CIM.

Proof. It is enough to prove that if $A_{1}$ and $A_{2}$ are two columns in $\mathbb{R}^{m}$, and neither $A_{1} \leq A_{2}$ nor $A_{2} \leq A_{1}$, then the inframodular function $u$ satisfies $u\left(A_{1}\right)+$ $u\left(A_{2}\right)>u\left(A_{1} \wedge A_{2}\right)+u\left(A_{1} \vee A_{2}\right)$.

The proof is in the Annex

Remark CIM and multidimensional majorization

\footnotetext{
${ }^{5}$ Each column represents an individual.
} 
It is worth noticing that the type of reduction of inequality or in other words of majorization envisioned in this paper is different from the usual multidimensional majorization. Actually, this feature is particularly clear when considering CIM (as observed by Zoli, 2009). Consider distributions $A$ and $B$ such that

$$
A=\left(\begin{array}{ll}
0 & 1 \\
1 & 0
\end{array}\right) \quad B=\left(\begin{array}{ll}
0 & 1 \\
0 & 1
\end{array}\right), \text { clearly } A>_{c} B
$$

but one cannot find any bistochastic matrix $\Pi=\left(\begin{array}{cc}\lambda & 1-\lambda \\ 1-\lambda & \lambda\end{array}\right)$ such that $\Pi B=A$.

The reader will find in Müller and Scarsini (2012) an extensive study of the sequence of inframodular transfers, which correspond to our type of majorization. This is performed in the case of general multidimensional probability measures. It would be interesting to derive the corresponding transfers in our simpler framework. This will be the subject of a future paper.

\section{Empirical Analysis}

Based on Theorem 3, we specialize $\psi(\cdot)$ as $\psi(\cdot)=\ln (\cdot)$, thus, considering cases in which all of the attributes are strictly positive we adopt $\forall A \in \mathcal{A}_{++}$as a 'mean' social evaluation function,

$$
J(A)=\frac{1}{n} \sum_{j=1}^{n} \ln \sum_{i=1}^{m} \alpha_{i} a_{i j}
$$

We aim at evaluating the pertinence of this inframodular function using data. In order to test it, we decided to make a comparison with another function that is not inframodular, namely the famous Human Development Index (HDI). This index was launched by United Nations in $1990 .{ }^{6}$ The classical version of this index basically works with three variables (life expectancy $(h)$, education $(e)$ and income $(w)$ ) and provides a value, which allows to obtain ranking among countries. Recently, in 2010, this index has been updated ${ }^{7}$ and now its form is,

$$
H(h, e, w)=h^{\frac{1}{3}} e^{\frac{1}{3}} w^{\frac{1}{3}}
$$

where each variable is an index between 0 and 1 and consists, basically, in the population mean, for example, $h=\frac{1}{n} \sum_{j=1}^{n} h_{j}$.

Besides the famous role of HDI, we can simply consider this index as a way to aggregate different attributes, as well. As $H(h, e, w)$ provides an outcome between 0 and 1 , we decided to extract the 'certainty equivalent' of $J(A)$, i.e., $I(A)=\exp ^{J(A)}$. Then, now we have both indexes providing results in the range $[0,1]$.

\footnotetext{
${ }^{6}$ For more details, see UNDP (1990).

${ }^{7}$ Zambrano (2014) has a discussion about this new index, its computation and axiomatization. See also Herrero et al. (2010).
} 
The database chosen is the Brazilian national exam for high school students. ${ }^{8}$ The final notes in this exam are split up in five categories: natural sciences, human sciences, languages, mathematics and essay writing. In order to apply $H(\cdot)$ in these data, we focused our analysis in only three attributes, namely, natural sciences $\left(a^{s}\right)$, languages $\left(a^{l}\right)$ and mathematics $\left(a^{m}\right)$.

The population $(n)$ is the number of students in each town. Following HDI rules, here we also give the same weight to the attributes. The function $J(A)$ in this case, writes as,

$$
J(A)=\frac{1}{n} \sum_{j=1}^{n} \ln \left(\frac{a_{j}^{s}+a_{j}^{l}+a_{j}^{m}}{3}\right)
$$

It is widely known that classical HDI formula does not consider in its calculation the level of inequality within a country. For this, a specific index is available called IHDI. ${ }^{9}$ However, we are interested in contrast with the classical HDI to detect in which extent $I(A)$ is influenced by the 'intra' inequalities. In other words, we want to see whether this function delivers a worst result for towns which have more inequality among their students. In this case, as the classical HDI neglects inequality characteristics, this comparison could be a good option to test the effectiveness of this function with respect to inequality.

Once Brazil has 5570 towns, then we confined our analysis to Minas Gerais state. Below the descriptive statistics are presented.

Table 1 - Descriptive Statistics

\begin{tabular}{lcccc}
\hline Variable & Mean & $\begin{array}{c}\text { Standard } \\
\text { Deviation }\end{array}$ & Minimum & Maximum \\
\hline Science & 0.49611 & 0.07456 & 0.34200 & 0.87640 \\
Language & 0.51850 & 0.06725 & 0.30620 & 0.79440 \\
Math & 0.49559 & 0.11077 & 0.31850 & 0.97360 \\
\hline Number of Students & \multicolumn{5}{c}{677,127} \\
\hline \multicolumn{2}{c}{ Results by Town } \\
\hline Function & Mean & Standard & Minimum & Maximum \\
\hline$I(A)$ & Deviation & 0,43577 & 0,53025 \\
$H(A)$ & 0.48412 & 0,01851 & 0.43748 & 0.53450 \\
\hline Number of Towns & 0.48765 & 0.01913 & 053 & \\
\hline
\end{tabular}

Firstly, the difference between the $I(A)$ and $H(A)$ outcomes is relatively small. Their correlation coefficient is 0,999 . The similarity of the outcomes is

\footnotetext{
${ }^{8}$ This exam is called ENEM (Exame Nacional do Ensino Médio - National high school exam). This exam is non-mandatory and has been used both as an admission test for enrollment in federal universities and educational institutes, as well as for certification for a high school degree.

${ }^{9}$ Kovacevic (2010) offers a good review and discussion about the importance of the inequality to evaluate the human development.
} 
suitable, because it shows that this function provides the outcomes in a similar sense as HDI usually does. Nevertheless, we may see through the descriptive statistics table that there are some differences between both functions' results and we are interested in them.

For example, despite the strong closeness among the outcomes, we found that $H(A)$ is always bigger than $I(A)$ for every town, and this difference varies. Thus, since HDI does not consider inequality in its computation, we would like to know if the size of the difference between the functions is related with inequality level of the towns. In other words, we want to see whether inequality is positively correlated with $H(A)-I(A)$. To measure the inequality in this case, we summed the values of the attributes for each student and extracted the standard deviation of this transformed variable. We want to analyze the relation between these two variables in order to assert whether $I(A)$ takes inequality into account or not.

To answer this question, we need to evaluate these variables jointly. Below in Figure 1, one will find the dispersion graph of these two variables.

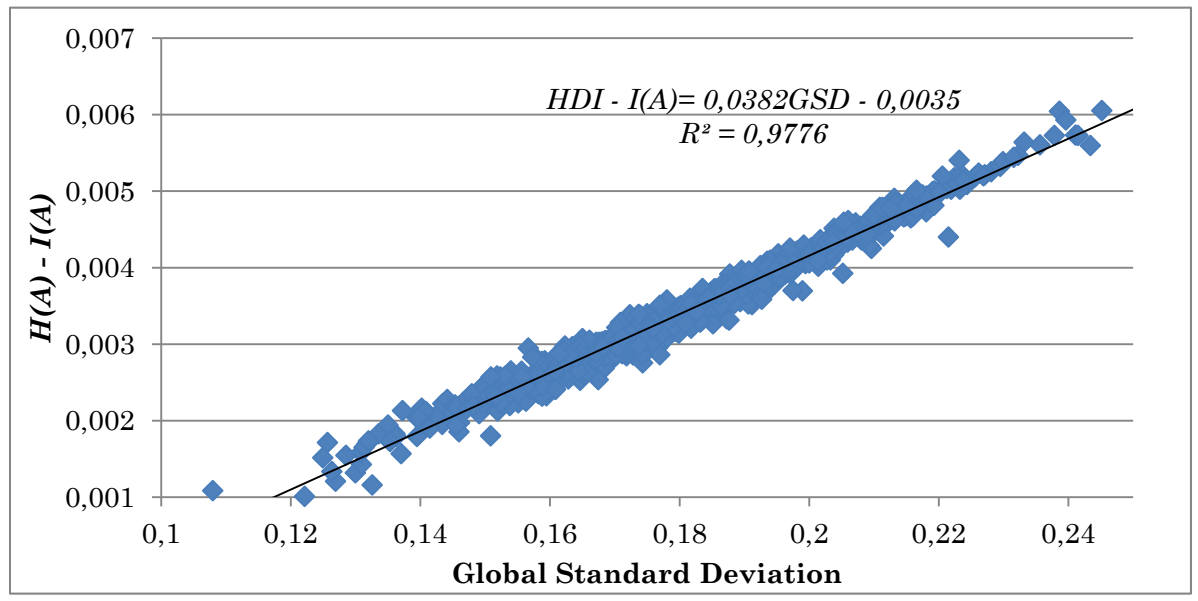

Figure 1: Dispersion graph between $H(A)-I(A)$ and the global standard deviation of the attributes

We, also, computed a linear regression as a hypothetical exercise. The equation is written in the graph and depicted by the black line. The value of $R^{2}$ attests that standard deviation explains almost $98 \%$ of the $H(A)-I(A)$ behavior. The positive relation between the variables is quite substantial.

Therefore, based on these results we suggest that this function could be a good alternative to IHDI. In short, we provide an inframodular function which can be used to aggregate several attributes (with different weights, if necessary), and takes into account the inequality inside the analyzed 'population'. 


\section{Concluding remarks}

This paper aimed at characterizing a simple 'additive' social evaluation function based on a particular type of inframodular function proposed by Müller and Scarsini. In the multidimensional case it allows to respect what can be considered a natural Pigou-Dalton principle. Furthermore, if the policy-maker aims at treating every individual equally, which might be fair, our social evaluation functions agree with the property of correlation increasing majorization, already suggested by Tsui.

Building upon a long tradition, we specify our functions in order to obtain a simple tractable relative inequality index. Finally we propose an empirical analysis aiming at evaluating the pertinence of a specific inframodular evaluation function à la Müller and Scarsini, when compared to the famous HDI functional.

\section{References}

Allison, R. A. and Foster, J. E. (2004). Measuring health inequality using qualitative data. Journal of health economics, 23(3):505-524.

Atkinson, A. B. (1970). On the measurement of inequality. Journal of economic theory, 2(3):244-263.

Atkinson, A. B. (1987). On the measurement of poverty. Econometrica, $55(4): 749-764$.

Atkinson, A. B. and Bourguignon, F. (1982). The comparison of multidimensioned distributions of economic status. The Review of Economic Studies, 49(2):183-201.

Boland, P. J. and Proschan, F. (1988). Multivariate arrangement increasing functions with applications in probability and statistics. Journal of Multivariate Analysis, 25(2):286-298.

Bosmans, K., Lauwers, L., and Ooghe, E. (2009). A consistent multidimensional Pigou-Dalton transfer principle. Journal of Economic Theory, 144(3):13581371.

Bourguignon, F. and Chakravarty, S. R. (2003). The measurement of multidimensional poverty. The Journal of Economic Inequality, 1(1):25-49.

Debreu, G. (1960). Topological methods in cardinal utility theory. In In K. J. Arrow, S. K. and Suppes, P., editors, Mathematical methods in the social sciences., pages 16-26. Stanford University Press, Stanford. CA.

Duclos, J.-Y., Sahn, D. E., and Younger, S. D. (2006). Robust multidimensional poverty comparisons. Economic Journal, 116(514):943-968.

Foster, J., Greer, J., Thorbecke, E., et al. (1984). A class of decomposable poverty measures. Econometrica, 52(3):761-66. 
Gajdos, T. and Weymark, J. A. (2005). Multidimensional generalized gini indices. Economic Theory, 26(3):471-496.

Galichon, A. and Henry, M. (2012). Dual theory of choice with multivariate risks. Journal of Economic Theory, 147(4):1501-1516.

Herrero, C., Martínez, R., and Villar, A. (2010). Multidimensional social evaluation: an application to the measurement of human development. Review of Income and Wealth, 56(3):483-497.

Kolm, S.-C. (1976a). Unequal inequalities. I. Journal of Economic Theory, $12(3): 416-442$.

Kolm, S.-C. (1976b). Unequal inequalities. II. Journal of Economic Theory, 13(1):82-111.

Kolm, S.-C. (1977). Multidimensional egalitarianisms. The Quarterly Journal of Economics, 91(1):1-13.

Kovacevic, M. (2010). Measurement of inequality in human development-a review, 2010/35. Human Development Research Papers (2009 to present), Human Development Report Office (HDRO), United Nations Development Programme (UNDP).

Maasoumi, E. (1986). The measurement and decomposition of multidimensional inequality. Econometrica, 54(4):991-97.

Marinacci, M. and Montrucchio, L. (2005). Ultramodular functions. Mathematics of Operations Research, 30(2):311-332.

Müller, A. and Scarsini, M. (2001). Stochastic comparison of random vectors with a common copula. Mathematics of operations research, 26(4):723-740.

Müller, A. and Scarsini, M. (2012). Fear of loss, inframodularity, and transfers. Journal of Economic Theory, 147(4):1490-1500.

Richard, S. F. (1975). Multivariate risk aversion, utility independence and separable utility functions. Management Science, 22(1):12-21.

Sen, A. (1976). Poverty: An ordinal approach to measurement. Econometrica, 44(2):219-231.

Shaked, M. (1982). A general theory of some positive dependence notions. Journal of Multivariate Analysis, 12(2):199-218.

Tsui, K.-Y. (1995). Multidimensional generalizations of the relative and absolute inequality indices: the atkinson-kolm-sen approach. Journal of Economic Theory, 67(1):251-265.

Tsui, K.-Y. (1999). Multidimensional inequality and multidimensional generalized entropy measures: An axiomatic derivation. Social Choice and Welfare, 16(1):145-157. 
UNDP (1990). Human development report. United Nations.

Weymark, J. A. (1981). Generalized gini inequality indices. Mathematical Social Sciences, 1(4):409-430.

Zambrano, E. (2014). An axiomatization of the human development index. Social Choice and Welfare, 42(4):853-872.

Zoli, C. (2009). From unidimensional to multidimensional inequality, welfare and poverty measurement. Technical report, Citeseer.

\section{Annex}

Marinacci and Montrucchio (2005) provided a through analysis of 'ultramodular functions', thus (by reversing the inequality in the definition) of what Müller and Scarsini (2012) called 'inframodular functions'.

Definition A function $f: \mathbb{R}^{n} \rightarrow \mathbb{R}$ is said to be inframodular if its increments are decreasing, namely:

(6) $\quad f(x+h)-f(x) \geq f(y+h)-f(y)$

for all $x, y \in \mathbb{R}^{n}$ with $x \leq y$ and $h \in \mathbb{R}^{n}, h \geq 0$

We intend now to prove that inframodular functions agree with our PigouDalton regressive transfers (see Introduction).

Lemma 1 if $f: \mathbb{R}^{n} \rightarrow \mathbb{R}$ is inframodular then $f$ satisfies Property 7 below:

(7) $\quad f(x)-f(x-h) \geq f(y+h)-f(y)$

for all $x, y \in \mathbb{R}^{n}$ with $x \leq y$ and $h \in \mathbb{R}^{n}, h \geq 0$

Proof. Let $x, y \in \mathbb{R}^{n} x \leq y$ and $h \geq 0$. Set $x^{\prime}=x-h$ and $y^{\prime}=y, h^{\prime}=h$ so $x^{\prime} \leq y^{\prime}$ and $h^{\prime} \geq 0$, therefore

(6) $\Longrightarrow f\left(x^{\prime}+h\right)-f\left(x^{\prime}\right) \geq f\left(y^{\prime}+h\right)-f\left(y^{\prime}\right)$

i.e. $f(x)-f(x-h) \geq f(y+h)-f(y)$

Lemma 2 (Proof of Theorem 4)

First, it is known that if $f$ is inframodular, then $f$ is submodular, i.e.,

$\forall a, b \in \mathbb{R}^{m} \quad f(a)+f(b) \geq f(a \wedge b)+f(a \vee b) \quad$ (see e.g. Marinacci and Montrucchio, 2005).

Let us show it again for sake of completeness.

Let $x=a \wedge b$, so $a=a \wedge b+h$ with $h \geq 0$.

Let $y=b$ one has $x \leq y$ and $h \geq 0$ so $f$ inframodular implies

$f(x+h)-f(x) \geq f(y+h)-f(y)$

$f(a)-f(a \wedge b) \geq f(b+a-a \wedge b)-f(b)$

but $b+a-a \wedge b=a \vee b$; hence, the result:

$f(a)+f(b) \geq f(a \wedge b)+f(a \vee b)$.

Thus one has $u\left(A_{1}\right)+u\left(A_{2}\right) \geq u\left(A_{1} \vee A_{2}\right)+u\left(A_{1} \wedge A_{2}\right)$

Since by hypothesis neither $A_{1} \leq A_{2}$ nor $A_{2} \leq A_{1}, u$ strict inframodular implies $u\left(A_{1}\right)+u\left(A_{2}\right)>u\left(A_{1} \vee A_{2}\right)+u\left(A_{1} \wedge A_{2}\right)$

Actually since not $A_{2} \leq A_{1}$, we get $A_{1} \wedge A_{2}<A_{2}$ so letting $x=A_{1} \wedge A_{2}$, $y=A_{2}, \xi=A_{1}-A_{1} \wedge A_{2}$, we get: 
$u(x+\xi)+u(y)>u(y+\xi)+u(x)$ i.e. $u\left(A_{1}\right)+u\left(A_{2}\right)>u\left(A_{1} \vee A_{2}\right)+u\left(A_{1} \wedge A_{2}\right)$.

\section{Appendix}

\section{Theorem 1}

Proof. We discuss only the sufficiency part, since the necessary proof is immediate.

From A.1, A.2, A.3, A.4 (weak order, continuity, monotonicity and independence) and $n \geq 3$, Theorem 3 in Debreu (1960) implies that there exist $n$ increasing and continuous functions $u_{j}: \mathbb{R}^{m} \longrightarrow \mathbb{R}$ such that $A \succsim B \Longleftrightarrow$ $\sum_{j=1}^{n} u_{j}\left(A_{j}\right) \geq \sum_{j=1}^{n} u_{j}\left(B_{j}\right)$ where the $u_{j}$ are unique up to affine transformation $\alpha u_{j}+\beta_{j}$ with $\alpha>0$ and $\beta_{j} \in \mathbb{R}$. Thus, we can assume that $\forall j u_{j}(0)=0$. From $A .5$ (anonymity), let us see that we can assume that there exists $u: \mathbb{R}^{m} \longrightarrow \mathbb{R}$, increasing and continuous such that: $A \succsim B \Longleftrightarrow \sum_{j=1}^{n} u\left(A_{j}\right) \geq \sum_{j=1}^{n} u\left(B_{j}\right)$. So, fix the $u_{j}$ such that $u_{j}(0)=0 \forall j$. By symmetry, we just need to prove that $u_{1}=u_{2}$. Take any $A_{1} \in \mathbb{R}^{m}$ and consider $\left(A_{1}, 0, A_{3}, \ldots, A_{n}\right)$ and $\left(0, A_{1}, A_{3}, \ldots, A_{n}\right)$. Through A.5: $u_{1}\left(A_{1}\right)+u_{2}(0)+\sum_{j=3}^{n} u_{j}\left(A_{j}\right)=u_{1}(0)+u_{2}\left(A_{1}\right)+\sum_{j=3}^{n} u_{j}\left(A_{j}\right)$, this entails straightforwardly $u_{1}\left(A_{1}\right)=u_{2}\left(A_{1}\right)$; thus, $u_{1}=u_{2}=\ldots=u_{n}=u$. So there exists $u: \mathbb{R}^{m} \longrightarrow \mathbb{R}$ increasing continuous (satisfying $u_{j}(0)=0$ ) such that $A \succsim B \Longleftrightarrow \sum_{j=1}^{n} u\left(A_{j}\right) \geq \sum_{j=1}^{n} u\left(B_{j}\right)$. Clearly, $u$ is defined up to a positive affine transformation 